Urszula Foryś and Zuzanna Szymańska (Warszawa)

\title{
MODELS OF INTERACTIONS BETWEEN HETEROTROPHIC AND AUTOTROPHIC ORGANISMS
}

Abstract. We present two simple models describing relations between heterotrophic and autotrophic organisms in the land and water environments. The models are based on the Dawidowicz \& Zalasiński models but we assume the boundedness of the oxygen resources. We perform a basic mathematical analysis of the models. The results of the analysis are complemented by numerical illustrations.

1. Introduction. In this paper we study two simple three-variable models of complex interactions between heterotrophic and autotrophic organisms depending on the amount of oxygen available in the environment. The models differ depending on the assumed environment - it can be either land or water. The basic models were proposed in [2], which is part of the project of mathematical modelling of the life phenomenon [1,2]. Such models are well known in the biomathematical literature (cf. e.g. [5]), but the Dawidowicz \& Zalasiński models differ from the standard ones in that the right-hand sides of the systems of equations involved are not smooth in general - there are some threshold values of the model parameters and variables which divide the phase space into subspaces where the right-hand sides are smooth. Therefore, mathematical analysis of these models requires a partition of the phase space into subspaces where each of the models is described by a standard three-variable ODE with a smooth right-hand side. The basic analysis of the model for the land environment was presented in [4].

In [4] we have also proposed a modification of the model for the land environment in which we assume that oxygen atoms are either bounded in diatomic molecules $\mathrm{O}_{2}$ or in carbon dioxide $\mathrm{CO}_{2}$ (i.e. we assume the

2000 Mathematics Subject Classification: 34C60, 34D05, 34D23, 92D40.

Key words and phrases: heterotrophic organism, autotrophic organism, ODEs, steady state, stability, global stability. 
boundedness of the oxygen resources). In our opinion such a modification captures the key aspects of the process of photosynthesis in a better way. Hence, we would like to propose the same modification for the model in the water environment.

In the present paper we focus on mathematical and numerical analysis of the modified models for both environments.

1.1. Presentation of the Dawidowicz \& Zalasinski models. In the model the following three variables are used: $x(t)$ and $y(t)$ describe the biomass of heterotrophic (animals) and autotrophic (green plants) organisms, respectively, and $z(t)$ is the volume of oxygen. For the land environment the model in [2] is described by

$$
\begin{aligned}
& \dot{x}=a x \min \left\{y,(z-K)^{+}\right\}-b x, \\
& \dot{y}=c y(M-y)-d x \min \left\{y,(z-K)^{+}\right\}, \\
& \dot{z}=e y(M-y)-f x \min \left\{y,(z-K)^{+}\right\},
\end{aligned}
$$

where $(z-K)^{+}=\max \{z-K, 0\}$ and all coefficients are positive.

In the first equation, which describes the dynamics of animal biomass, the first term, ax $\min \left\{y,(z-K)^{+}\right\}$, is responsible for the animal reproduction, which is proportional to their density (number of animals) and the minimum of the following two quantities: the density of autotrophic organisms and the volume of the accessible oxygen above some minimal threshold (denoted by the constant $K$ ). Using such a reproduction term, the authors intend to account for the fact that animals cannot survive in an environment with both poor nutrient supply and poor oxygen supply. The second term, $-b x$, corresponds to natural death of animals.

In the second equation, describing the dynamics of green plant biomass, the first term, $c y(M-y)$, denotes its growth, which is limited by the capacity of the environment, denoted by the constant $M$ (the logistic term is used to model that fact). The second term, $-d x \min \left\{y,(z-K)^{+}\right\}$, describes the decline of plants due to animal consumption, which is proportional to the animal biomass and the minimum of two quantities: the plant biomass and the oxygen volume (again the authors assume that the animals can only survive if there is enough oxygen, i.e. above the threshold $K$ ).

In the third equation, describing the changes in oxygen volume, the first term (analogous to the first term in the second equation), ey $(M-y)$, denotes the increase of the oxygen volume because of its production by green plants. The second term (again analogous to the second term in the second equation), $-f x \min \left\{y,(z-K)^{+}\right\}$, describes the decline of the oxygen volume because of animal activity, which is again proportional to the animal biomass and the minimum of the plant biomass and oxygen volume. 
A slightly different system of equations is proposed to describe the same processes in the water environment:

$$
\begin{aligned}
& \dot{x}=a x \min \{y, z, N\}-b x, \\
& \dot{y}=c y(M-y)-d x \min \{y, z, N\}, \\
& \dot{z}=e y(M-y)-f x \min \{y, z, N\}-g(z-N)^{+},
\end{aligned}
$$

with positive coefficients and the same meaning of the term $(z-N)^{+}$as before.

The main difference between the models for the land and water environments is that the concentration of oxygen diluted in water is limited, and its decrease is associated not only with consumption but also with the escape of oxygen surplus which was not diluted.

In the first equation, corresponding to animal biomass, the first term, ax $\min \{y, z, N\}$, describes the growth of animal population, which is again proportional to their concentration and the minimum of: the plant biomass, the accessible oxygen and the maximal amount of oxygen that can be diluted in water (denoted by the constant $N$ ). The second term, $-b x$, as previously, corresponds to natural death of animals.

In the second equation, describing the dynamics of green plant biomass, the first term, $c y(M-y)$, reflecting its growth is the same as in the model for the land environment, while the second one, $-d x \min \{y, z, N\}$, describing the decline of plants due to animal consumption, is (analogously to the previous equation) proportional to the animal biomass and the minimum of: the plant biomass, the accessible oxygen and the maximal amount of oxygen that can be diluted in water.

In the third equation, describing the changes in accessible oxygen in water, the first term (analogous to the first term in the second equation), $e y(M-y)$, stands for the production of oxygen by green plants during the process of photosynthesis. The second term (again analogous to the second term in the second equation), $-f x \min \{y, z, N\}$, is the decline of oxygen due to animal consumption. The additional third term, $-g(z-N)^{+}$, describes the escape of non-diluted oxygen surplus.

1.2. Properties of the Dawidowicz $\&$ Zalasinski models. As mentioned in [2] and in the introduction, the models can be analysed separately in different subspaces of the phase space in which the right-hand sides of (1.1) and (1.2) are smooth. Such analysis for (1.1) was performed in [4]. In this subsection we summarise the results of that analysis and make some remarks concerning the second model described by (1.2).

In [2] it was proved that:

1. The phase space for both models can be restricted to the set $\mathcal{D}=$ $\{(x, y, z): x>0,0<y<M, z>0\}$. 
2. The last variable $z$ can be expressed as a functional of $y$, where

$$
z(t)=z_{0}+\frac{f}{d}\left(y(t)-y_{0}\right)+\left(e-\frac{c f}{d}\right) \int_{0}^{t} y(s)(M-y(s)) d s,
$$

for (1.1) and (1.2) with $z \leq N$, while for (1.2) and $z>N$ this functional has the following form:

$$
\begin{aligned}
z(t)= & N+\left(z_{0}-M-\frac{f}{d} y_{0}\right) \mathrm{e}^{-g t} \\
& +\frac{f}{d} y(t)+\int_{0}^{t} y(s)\left(M\left(e-\frac{c f}{d}\right)-\frac{f g}{d}-y(s)\right) \mathrm{e}^{g(s-t)} d s .
\end{aligned}
$$

The authors of [2] also remarked that in the case of optimal level of oxygen both models can be simplified to the well-known predator-prey model with carrying capacity for preys (see e.g. [5] or [3]). Clearly, if $z>y+K$ in the land environment or $y<z \leq N$ in water, then the predator-prey system for $x$ and $y$ is uncoupled with $z$, and $z$ can be calculated from (1.3) for both equations (1.1) and (1.2). This is the only case when both (1.1) and (1.2) have the same form. Similarly to [4] we can prove the following properties:

I. If $b / a>M, e>c, d>f$, then

- for $z_{0}>y_{0}+K$, the solution to (1.1) stays in the subregion $\mathcal{D}_{1}^{1}=$ $\{(x, y, z) \in \mathcal{D}: z>y+K\}$

- for

$$
y_{0}<z_{0}<N-\frac{e}{c}\left(M-y_{0}\right)-x_{0} \frac{M}{b-a M}\left(\frac{e d}{c}-f\right)<N,
$$

the solution to (1.2) stays in $\mathcal{D}_{1}^{2}=\{(x, y, z) \in \mathcal{D}: N>z>y\}$.

Moreover, $(x, y, z) \rightarrow\left(0, M, z_{g}\right)$ where

$$
z_{g}=z_{0}+\frac{f}{d}\left(M-y_{0}\right)+\left(e-\frac{c f}{d}\right) \int_{0}^{\infty} y(s)(M-y(s)) d s .
$$

II. If $b / a<M, x_{0}<(e / f)\left(M-y_{0}\right), e>c$ and $d>f$, then $(x, y) \rightarrow$ $((c / d)(M-b / a), b / a)$ and $z$ increases. Therefore, for (1.1) the solution stays in $\mathcal{D}_{1}^{1}$ and $z \rightarrow \infty$, while the solution to (1.2) leaves $\mathcal{D}_{1}^{2}(z>N$ at some time).

In the case of non-optimal level of oxygen, equations (1.1) and (1.2) have different forms. In [4] for (1.1) it was proved that:

- If $0<z_{0}<K-(e / c)\left(M-y_{0}\right)$, then the solution to (1.1) stays in $\mathcal{D}_{2}^{1}=\{(x, y, z) \in \mathcal{D}: z<K\}$ and $(x, y, z) \rightarrow\left(0, M, z_{0}+(e / c)\left(M-y_{0}\right)\right)$.

- If $(e / c)\left(M-y_{0}\right)<z_{0}<K$, then the solution to (1.1) leaves $\mathcal{D}_{2}^{1}$. 
- If $K<z_{0}<y_{0}+K, e<c$ and $d<f$, then the solution to (1.1) stays in $\mathcal{D}_{3}^{1}=\{(x, y, z) \in \mathcal{D}: K<z<y+K\}$.

Similarly, we can prove the following properties of the solutions to (1.2):

- If $b / a<M<N, y_{0}<N<z_{0}$ and $x_{0}<(e / f)\left(M-y_{0}\right)$, then the solution stays in $\mathcal{D}_{2}^{2}=\{(x, y, z) \in \mathcal{D}: y<N<z\}$.

- If $M>N$ and $y_{0}<N<z_{0}$, then there exists $\bar{t}>0$ such that the solution leaves $\mathcal{D}_{2}^{2}$ at $\bar{t}$.

- If $M>N>b / a, y_{0}>N$ and $z_{0}>N$, then there exists $\bar{t}>0$ such that the solution leaves $\mathcal{D}_{3}^{2}=\{(x, y, z) \in \mathcal{D}: y>N$ and $z>N\}$.

- If $M<N$, ed $<c f, f<d$ and $y_{0}>(d / f) z_{0}$, then the solution stays in $\mathcal{D}_{4}^{2}=\{(x, y, z) \in \mathcal{D}: y>z$ and $z<N\}$.

- If $z_{0}<y_{0}, z_{0}<N$ and $z_{0}+(e / c)\left(M-y_{0}\right)$, then the solution leaves $\mathcal{D}_{4}^{2}$.

The proofs of the above properties of (1.2) are similar to those given in [4] for (1.1). We include part of them in the Appendix.

2. Modified models. In our opinion the system needs some modifications. Oxygen is produced by autotrophs from carbon dioxide in the photosynthesis process. On the other hand, oxygen is consumed by heterotrophs, with carbon dioxide as one of the products. Therefore, we assume that the common amount of oxygen atoms in the atmosphere is constant and equal to some parameter $L$. Hence, we propose a linear dependence of the oxygen production on the autotrophs density with coefficient proportional to $L-z$. Finally, under the assumptions above, the system for land environment takes the form

$$
\begin{aligned}
& \dot{x}=a x \min \left\{y,(z-K)^{+}\right\}-b x, \\
& \dot{y}=c y(M-y)-d x \min \left\{y,(z-K)^{+}\right\}, \\
& \dot{z}=e y(L-z)-f x \min \left\{y,(z-K)^{+}\right\} .
\end{aligned}
$$

In the modified system a positive steady state exists and it can be stable (cf. [4] where examples of stable behaviour of the modified system were shown). We observe quick stabilisation of the oxygen level and the balance between heterotrophs and autotrophs. In our opinion this type of system dynamics reflects natural processes better than in the case of the original system described by (1.1).

Similarly, after modification, the system for the water environment has the form

$$
\begin{aligned}
& \dot{x}=a x \min \{y, z, N\}-b x, \\
& \dot{y}=c y(M-y)-d x \min \{y, z, N\}, \\
& \dot{z}=e y(L-z)-f x \min \{y, z, N\}-g(z-N)^{+} .
\end{aligned}
$$


2.1. Properties of the modified systems. The Dawidowicz \& Zalasiński models and those proposed in [4] and in this paper differ only in the third equation. Therefore, under some assumptions their properties may be similar. However, note that after the proposed modification the density $z(t)$ of oxygen does not increase unboundedly, as is possible in the case of the original model in the land environment. Also note that in the general case for the modified systems described by (2.1) and (2.2) there is no formula for $z$ as a functional of $y$ (like formulae (1.3) or (1.4) for equations (1.1) and (1.2)).

It is easy to see that the modified systems can be analysed in the phase space $\mathcal{A}=\{(x, y, z): x>0,0<y<M, 0<z<L\}$. Hence, we restrict our analysis to this space.

Properties of (2.1). For the system defined by (2.1) we have three cases:

(a) $z<K$;

(b) $z>K$ and $\min \left\{y,(z-K)^{+}\right\}=y$;

(c) $z>K$ and $\min \left\{y,(z-K)^{+}\right\}=z-K$.

In case (a) the dynamics of solutions is very simple because the equations for $x$ and $y$ are uncoupled.

LEMMA 1. If $z_{0}<K$ and $L \leq K$, then the solution to (2.2) stays in the subregion $\mathcal{A}_{1}^{1}=\{(x, y, z) \in \mathcal{A}: z<K\}$, while for $L>K$ it leaves $\mathcal{A}_{1}^{1}$.

Proof. Until $z<K$ we have $\min \left\{y,(z-K)^{+}\right\}=0$. Hence, $\dot{x}=-b x$ and the dynamics of $y$ is described by the well-known logistic equation $\dot{y}=$ $c y(M-y)$ (proposed by Verhulst in [6]). Therefore, $x \rightarrow 0$ exponentially and $y \rightarrow M$ as a solution to the logistic equation (see e.g. [5] or [3]). In this case it is possible to express $z$ as a functional of $y$. Clearly, dividing the second equation of (2.1) by the third, one gets

$$
\frac{d z}{d y}=\frac{c}{e} \frac{L-z}{M-y},
$$

which implies

$$
z(t)=L-\left(L-z_{0}\right)\left(\frac{M-y(t)}{M-y_{0}}\right)^{c / e} .
$$

Hence, $z$ increases to $L$ as $y$ increases to $M$.

Finally, if $L \leq K$, then $z(t)<K$ for every $t \geq 0$, while for $L>K$ there exists $\bar{t}>0$ such that $z(\bar{t})=K$ and the solution to (2.1) leaves $\mathcal{A}_{1}^{1}$.

In case (b) the first and second equations of (2.1) form the predatorprey system (in $(x, y)$ coordinates) with carrying capacity for preys (see e.g. [5] or [3]) whose dynamics depends on the magnitude of $M$. In this case the inequality $y<z-K$ is satisfied so we are interested in the subregion $\mathcal{A}_{2}^{1}=\{(x, y, z) \in \mathcal{A}: z>K+y\}$. 
Lemma 2. If $M<b / a, L>K+M, c / d>e / f, y_{0}<L-K-(f / e) x_{0}$ and $z_{0}>K+y_{0}$, then $x \rightarrow 0, y \rightarrow M, z \rightarrow L$ and the solution to (2.1) stays in $\mathcal{A}_{2}^{1}$.

Proof. If $M<b / a$ and $0<y_{0}<M$, then $x$ is decreasing to 0 and $y \rightarrow M$. Moreover, $y$ takes the minimal value on the isocline $y=M-(d / c) x$ (see e.g. [5]). If the solution stays in $\mathcal{A}_{2}^{1}$, then $\dot{z}(t)=e y(t)(L-z(t))-f x(t) y(t)$ for every $t \geq 0$. Therefore, for every $\varepsilon>0$ there exists $\bar{t}>0$ such that

$$
e(M-\varepsilon)(L-z(t))-M \varepsilon<\dot{z}(t)<e M(L-z(t)) \quad \text { for } t>\bar{t} .
$$

Asymptotically one gets $L \leq z \leq L+M \varepsilon / e(M-\varepsilon)$ for every $\varepsilon>0$, which yields $z \rightarrow L$ as $t \rightarrow \infty$.

In $\mathcal{A}_{2}^{1}$ the inequality $z(t)>y(t)+K$ for $t \geq 0$ must be satisfied. If the solution leaves $\mathcal{A}_{2}^{1}$, then there exists $\widetilde{t}>0$ such that $z(\widetilde{t})=y(\widetilde{t})+K$. The straight line $y=L-K-(f / e) x$ lies above the isocline $y=M-(d / c) x$ in the $(x, y)$ plane, and therefore the inequality $y<L-K-(f / e) x$ is satisfied under the assumptions of this lemma. Hence, $\dot{z}(\widetilde{t})=y(\widetilde{t})(e(L-K-y(\widetilde{t}))-$ $f x(\widetilde{t}))>0$, which guarantees that the solution stays in $\mathcal{A}_{2}^{1}$.

If $M>b / a$, then solutions to predator-prey systems with carrying capacity for preys are non-monotonic. Moreover, there exists a positive steady state $(\bar{x}, \bar{y})=((c / d)(M-b / a), b / a)$, which is globally stable in $\left(\mathbb{R}^{+}\right)^{2}$. Hence, if the solution to (2.1) stays in $\mathcal{A}_{2}^{1}$, then we can estimate the growth of $z$.

Lemma 3. If $M>b / a, L>K+M+\frac{c f}{d e}(M-b / a), c / d>e / f$ and $z_{0}>y_{0}+K$, then there exists a neighbourhood $\mathcal{U}$ of the positive steady state $(\bar{x}, \bar{y}) \in \mathcal{U}$ such that if $\left(x_{0}, y_{0}\right) \in \mathcal{U}$, then the solution to (2.1) stays in $\mathcal{A}_{2}^{1}$, $y(t)<L-K-(f / e) x(t)$ for $t \geq 0$ and $x \rightarrow \bar{x}, y \rightarrow \bar{y}$ and $z \rightarrow \bar{z}=L-(f / e) \bar{x}$.

Proof. Under the assumptions of this lemma the positive steady state in $(x, y)$ coordinates lies in the region $y<L-K-(f / e) x$. Due to its global asymptotic stability in the positive quadrant $\left(\mathbb{R}^{+}\right)^{2}$ (see e.g. [5]) there exists an open subset $\mathcal{U} \subset\left(\mathbb{R}^{+}\right)^{2}$ such that if $\left(x_{0}, y_{0}\right) \in \mathcal{U}$, then the solution stays in the region $y<L-K-(f / e) x$. If the solution stays in $\mathcal{A}_{2}^{1}$, then $z$ tends to $\bar{z}$, which can be shown similarly to the proof of Lemma 2 . However, the inequality $\bar{z}>\bar{y}+K$ is necessary in this case. We have

$$
\bar{z}=L-\frac{c f}{d e}\left(M-\frac{b}{a}\right)>M+K>\bar{y}+K
$$

under the assumptions of this lemma.

Finally, as in the proof of Lemma 2 we show that if $z(\bar{t})=y(\bar{t})+K$ for some $\bar{t}>0$, then $\dot{z}(\bar{t})>0$, which implies that the solution stays in $\mathcal{A}_{2}^{1}$.

In case (c) all three equations of (2.1) are coupled. Now, we study the dynamics of (2.1) in the subregion $\mathcal{A}_{3}^{1}=\{(x, y, z) \in \mathcal{A}: K<z<y+K\}$. 
As in case (b), the existence of a positive steady state depends on the model parameters.

Looking for steady states we get either $x=0$ or $z=\bar{z}=K+b / a$ from the first equation of (2.1). If $x=0$, then $y=0$ or $y=M$ from the second equation. However, only $y=M$ can be stable (asymptotically, if $x \rightarrow 0$, then $y$ tends to the solution of the logistic equation). Therefore, we are interested in the stability of the semi-trivial steady-state $(0, M, L)$. On the other hand, if $z=\bar{z}$, then

$$
e \bar{y}\left(L-K-\frac{b}{a}\right)=f \frac{b}{a} \bar{x}
$$

from the third equation of (2.1). This implies that the positive steady state exists only if $L>K+b / a$. Moreover, calculating $\bar{y}$ one gets

$$
\bar{y}=M-\frac{d e}{c f}\left(L-K-\frac{b}{a}\right),
$$

which yields

$$
M>\frac{d e}{c f}\left(L-K-\frac{b}{a}\right)
$$

Finally,

$$
\bar{x}=\frac{a e}{b f} \bar{y}\left(L-K-\frac{b}{a}\right) .
$$

Lemma 4. If $L<K+b / a$, then the semi-trivial steady state $(0, M, L)$ of (2.1) is locally asymptotically stable and the positive steady state does not exist.

If $L>K+b / a$ and $M>\frac{d e}{c f}(L-K-b / a)$, then there exists a positive steady state $(\bar{x}, \bar{y}, \bar{z}) \in \mathcal{A}_{3}^{1}$ and the semi-trivial steady state is unstable.

Proof. As shown above, the inequality $L>K+b / a$ is necessary for the existence of a positive steady state for (2.1). Hence, if the reverse inequality is satisfied, then the positive steady state does not exist. To study the local stability of the semi-trivial steady state we calculate the Jacobi matrix of the system

$$
J(x, y, z)=\left(\begin{array}{ccc}
a(z-K)-b & 0 & a x \\
-d(z-K) & c M-2 c y & -d x \\
-f(z-K) & e(L-z) & -e y-f x
\end{array}\right) .
$$

For the semi-trivial steady state this matrix is lower triangular and it is easy to see that its characteristic values are $\lambda_{1}=a(L-K)-b, \lambda_{2}=-c M$, $\lambda_{3}=-e M$. Hence, all of them are real and negative iff $L<K+b / a$.

The existence of a positive steady state was considered above. 
The stability of the positive steady state can be analysed using the Routh-Hurwitz criterion. Setting $u=L-\bar{z}=L-K-b / a$ one gets

$$
J(\bar{x}, \bar{y}, \bar{z})=\left(\begin{array}{ccc}
0 & 0 & \frac{a^{2} e}{b f}\left(M-\frac{d e}{c f} u\right) u \\
-\frac{b d}{a} & \frac{2 d e}{f} u-M c & -\frac{a d e}{b f}\left(M-\frac{d e}{c f} u\right) u \\
-\frac{b f}{a} & e u & -e\left(M-\frac{d e}{c f} u\right)\left(1+\frac{a}{b} u\right)
\end{array}\right) .
$$

Calculating the characteristic polynomial for this steady state,

$$
W(\lambda)=\lambda^{3}+A_{2} \lambda^{2}+A_{1} \lambda+A_{0},
$$

we see that

$$
A_{0}=\operatorname{ace}\left(M-\frac{d e}{c f} u\right)^{2} u>0
$$

and

$$
\begin{aligned}
A_{1}= & \frac{a d^{2} e^{3}}{c f^{2}} u^{3}+\frac{2 b d^{2} e^{3}-2 a c d e^{2} f M-a b d e^{2} f}{c f^{2}} u^{2} \\
& +\frac{\left(a c e f M+a b e f-3 b d e^{2}\right) M}{f} u+b c e M^{2}, \\
A_{2}= & -\frac{a d e^{2}}{c f} u^{2}+\frac{a c e f M-b d e^{2}-2 b c d e}{c f} u+b(c+e) M .
\end{aligned}
$$

Therefore, the Routh-Hurwitz criterion (see e.g. [5]) implies stability for small positive values of $u$ and instability for large $u$. Clearly, for $u \rightarrow 0$ (that is, $\bar{z} \rightarrow L)$ we have $A_{1} A_{2}-A_{0} \rightarrow b^{2} c e(c+e) M^{3}>0$, while for $u \rightarrow \infty$ we have $A_{1} A_{2}-A_{0} \rightarrow-\infty$. On the other hand, in our case positivity of the steady state yields $u<\frac{c f}{e d} M$. This suggests that the sign of $A_{1} A_{2}-A_{0}$ depends on the magnitude of $M$. However, for $u \rightarrow \frac{c f}{e d} M$ one gets

$$
A_{1} A_{2}-A_{0} \rightarrow \frac{a c^{2} f M^{4}}{d}\left(c^{2}-1\right)(3 a c f M+a b f-3 b d e-2 c b d),
$$

which shows that stability also depends on the magnitude of other parameters, mainly on $c$.

Corollary 1. For $L>K+b / a$ and $M>\frac{d e}{c f}(L-K-b / a)$, if $L-$ $K-b / a$ is sufficiently small, then the positive steady state $(\bar{x}, \bar{y}, \bar{z})$ is locally asymptotically stable in $\mathcal{A}_{3}^{1}$.

Properties of (2.2). For the second system defined by (2.2) the following four cases can be considered:
(A) $y<z<N$;
(B) $z<N$ and $z<y$;
(C) $z>N$ and $y>N$;
(D) $z>N>y$. 
In case $(A)$ the system (2.2) takes the same form as (2.1) in case (b), but the subregion where this form is valid is different from that for (2.1). Now we are interested in $\mathcal{A}_{1}^{2}=\{(x, y, z) \in \mathcal{A}: y<z<N\}$.

Lemma 5. If $M<b / a, N>L>M, c / d>e / f, y_{0}<L-(f / e) x_{0}$ and $z_{0}>y_{0}$, then $x \rightarrow 0, y \rightarrow M, z \rightarrow L$ and the solution to $(2.2)$ stays in $\mathcal{A}_{1}^{2}$.

If $M<b / a$ and $L<M$, then the solution leaves $\mathcal{A}_{1}^{2}$.

Proof. The proof of the first part is similar to the proof of Lemma 2.

The second part is obvious. If the solution stays in $\mathcal{A}_{1}^{2}$, then $z \rightarrow L$ and $y \rightarrow M$; but this implies $z(t)<y(t)$ for sufficiently large $t$, which contradicts the definition of $\mathcal{A}_{1}^{2}$.

If $M>b / a$, then every solution to (2.2) staying in $\mathcal{A}_{1}^{2}$ has the same limit as the solution to (2.1) in case (b), that is, $x \rightarrow \bar{x}=(c / d)(M-\bar{y})$, $y \rightarrow \bar{y}=b / a$ and $z \rightarrow \bar{z}=L-(f / e) \bar{x}$.

In case (B) all three equations of (2.2) are coupled as in case (c) for (2.1), but the form of (2.2) is slightly different from that of (2.1) in case (c) - the term $z-K$ is replaced by $z$. Therefore, there is the same semi-trivial steady state $(0, M, L)$, and the positive steady state

$$
(\bar{x}, \bar{y}, \bar{z})=\left(\frac{e}{f}\left(L-\frac{b}{a}\right)\left(M-\frac{d e}{c f}\left(L-\frac{b}{a}\right)\right), M-\frac{d e}{c f}\left(L-\frac{b}{a}\right), \frac{b}{a}\right)
$$

exists iff $M>\frac{d e}{c f}(L-b / a)>0$. We study the behaviour of the system in the subregion $\mathcal{A}_{2}^{2}=\{(x, y, z) \in \mathcal{A}: z<\min (N, y)\}$.

Lemma 6. If $L<b / a$ and $L<\min (N, M)$, then the semi-trivial steady state $(0, M, L) \in \mathcal{A}_{2}^{2}$ of $(2.2)$ is locally asymptotically stable and the positive steady state does not exist.

If $L>b / a, N>b / a$ and $M-\frac{d e}{c f}(L-b / a)>b / a$, then the positive steady state $(\bar{x}, \bar{y}, \bar{z}) \in \mathcal{A}_{2}^{2}$ exists and the semi-trivial steady state is unstable.

Proof. As in case (c) the Jacobi matrix is lower triangular and we can easily find the characteristic values $\lambda_{1}=a L-b, \lambda_{2}=-c M<0$ and $\lambda_{3}=-e \bar{y}<0$. Hence, all three characteristic values are real and negative iff $L<b / a$. If $L<b / a$, then $\bar{z}>L$, which contradicts the definition of the phase space $\mathcal{A}$. Thus, the positive steady state does not exist.

The first coordinate of the positive steady state $\bar{x}$ is positive iff $L>b / a$ and $M>\frac{d e}{c f}(L-b / a)$. Under the assumptions, $\bar{z}<N$ and $\bar{z}<\bar{y}$, which yields $(\bar{x}, \bar{y}, \bar{z}) \in \mathcal{A}_{2}^{2}$. This completes the proof.

To study local stability of the positive steady state one can see that the Jacobi matrix is exactly the same as in case (c) for (2.1). Therefore, the conditions of stability are also the same. 
In case (C) the interesting subregion is defined by $\mathcal{A}_{3}^{2}=\{(x, y, z) \in \mathcal{A}$ : $N<\min (y, z)\}$. In this case the first equation of $(2.2)$ is uncoupled and $x(t)=x_{0} \mathrm{e}^{(a N-b) t}$.

Lemma 7. If $N>b / a$, then the solution to (2.2) leaves $\mathcal{A}_{3}^{2}$.

If $N<b / a, N<\min (M, L), M>\frac{d e}{c f}(L-N)$ and

$$
x_{0}<\min \left(M-\frac{d e}{c f}(L-N), \frac{c}{d}(M-N)\right),
$$

$y_{0}<N$ and $z_{0}<N$, then the solution stays in $\mathcal{A}_{3}^{2}$ and

$$
x \rightarrow 0, \quad y \rightarrow M, \quad z \rightarrow \frac{e M L+g N}{e M+g} .
$$

Proof. If $N>b / a$, then $x \rightarrow \infty$ as $t \rightarrow \infty$, which implies $\dot{y} \rightarrow-\infty$. Therefore, there exists $\bar{t}>0$ such that $y(\bar{t})<N$ and the solution leaves $\mathcal{A}_{3}^{2}$.

If $N<b / a$, then $x$ decreases to 0 . This implies that $y \rightarrow M$ and if $x_{0}<(c / d)(M-N)$, then $y$ does not drop below $N$. Clearly, if there exists $\bar{t}>0$ such that $y(\bar{t})=N$, then $\dot{y}(\bar{t})=c N(M-N)-d N x(\bar{t})>c N(M-N)$ $-d N x_{0}>0$, which contradicts the definition of $\bar{t}$.

Similarly we show that $z \rightarrow \frac{e M L+g N}{e M+g}$ and $z(t)>N$ for every $t \geq 0$.

In the last case (D) we study the system in $\mathcal{A}_{4}^{2}=\{(x, y, z) \in \mathcal{A}: y<$ $N<z\}$. Then the first two equations of (2.2) form a predator-prey system and the dynamics depends on the magnitude of $M$.

Lemma 8. If $M<b / a, M<N<L, y_{0}<N<z_{0}$ and $x_{0}<(e / f)(L-N)$, then the solution to $(2.2)$ stays in $\mathcal{A}_{4}^{2}$ and tends to $(0, M, L)$.

If $M>b / a, M<N<L$ and $(c / d)(M-b / a)<(e / f)(L-N)$, then there exists a neighbourhood $\mathcal{U}$ of $((c / d)(M-b / a), b / a)$ in $(x, y)$ coordinates such that if $\left(x_{0}, y_{0}\right) \in \mathcal{U}$, then $x(t)<(e / f)(L-N)$ and the solution stays in $\mathcal{A}_{4}^{2}$ and tends to

$$
\left(\frac{c}{d}\left(M-\frac{b}{a}\right), \frac{b}{a}, \frac{b e L+a g N-\frac{b c f}{d}(M-b / a)}{b e+a g}\right) .
$$

Proof. It is similar to those presented above.

3. Numerical solutions and discussion. We now present some results of numerical simulations for systems (2.1) and (2.2). In Fig. 1 we see a typical example of solution and phase space portrait for (2.1) in the case described by Lemma 1 and analysed in the previous section. In this case, there is lack of oxygen, $z<K$, heterotrophs become extinct, autotrophs stabilise on their carrying capacity $M$ and oxygen stabilises on its maximal level $L$. 

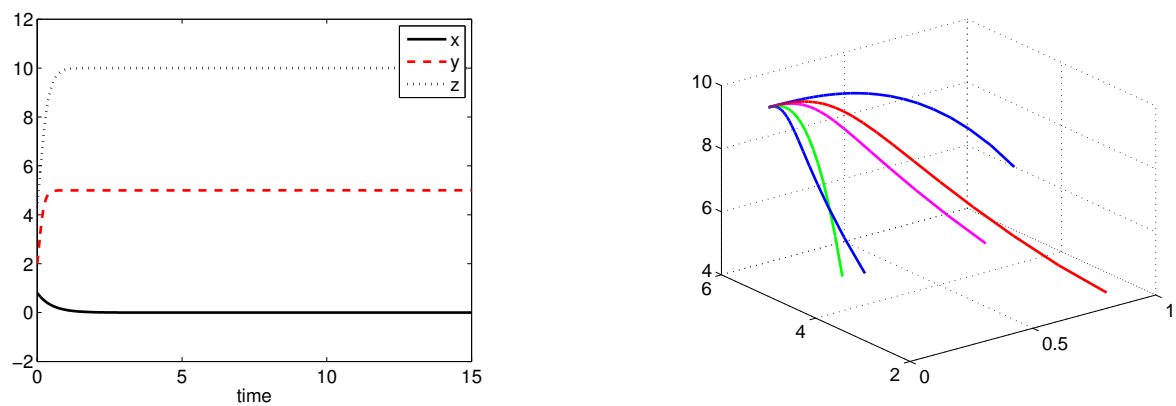

Fig. 1. Example of solution and phase space portrait for (2.1) in the first case of Lemma 1 with $z_{0}<K$ and $L \leq K$ (parameter values: $a=5, b=c=2, d=e=1, f=3, K=15$, $M=5$ and $L=10$ )
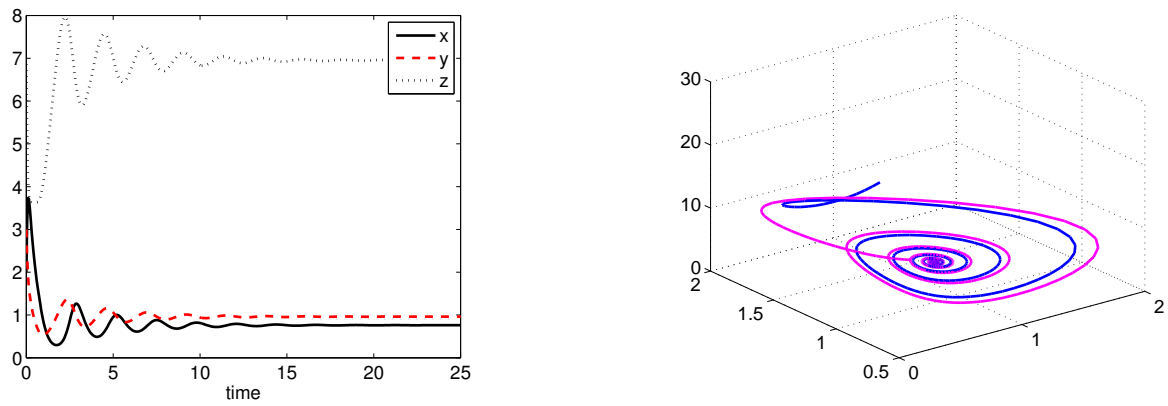

Fig. 2. Example of solution and phase space portrait for (2.1) in the case of Lemma 3 with $M>b / a$ and $L>K+M+\frac{c f}{d e}(M-b / a), c / d>e / f$ and $z_{0}>y_{0}+K$ (parameter values: $a=5.2, b=5, c=0.6, d=e=2, f=8, K=3, M=3.5$ and $L=10$ )

In Fig. 2 we see a typical example of solution and phase space portrait for (2.1) in the case described by Lemma 3. In this case, there is enough oxygen and typically, a positive steady state exists. The solutions stay in the initial subregion of the phase space and stabilise on the level of the positive steady state. This case can be interpreted as "normal" condition, the most common in nature in our opinion.

In Fig. 3 we present an example of solution and phase space portrait for (2.2) in the case described by Lemma 8. Similarly to Fig. 2 we observe stabilisation on the level of the positive steady state.

In Fig. 4 we present an example of solution that changes subregions of the phase space. We start from the subregion $\mathcal{A}_{2}^{1}$ with $M>b / a$. Initially, the solution in variables $x$ and $y$ behaves as in the predator-prey system with carrying capacity for preys (cf. [5] or [3]) until $z(t)<K$ when the solution switches to the subregion $\mathcal{A}_{1}^{1}$ where $x \rightarrow 0, y \rightarrow M, z \rightarrow L$. 

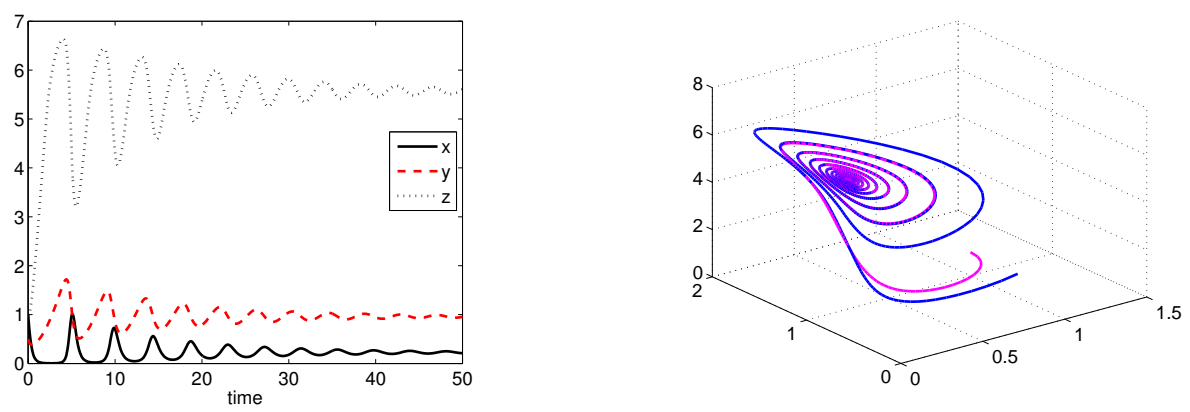

Fig. 3. Example of solution and phase space portrait for (2.2), the second case of Lemma 8 with $M>b / a, M<N<L$ and $(c / d)(M-b / a)<(e / f)(L-N)$ (parameter values: $a=5.2, b=5, c=0.15, d=2, e=1, f=8, g=1, K=3, M=4, L=8$ and $N=5)$
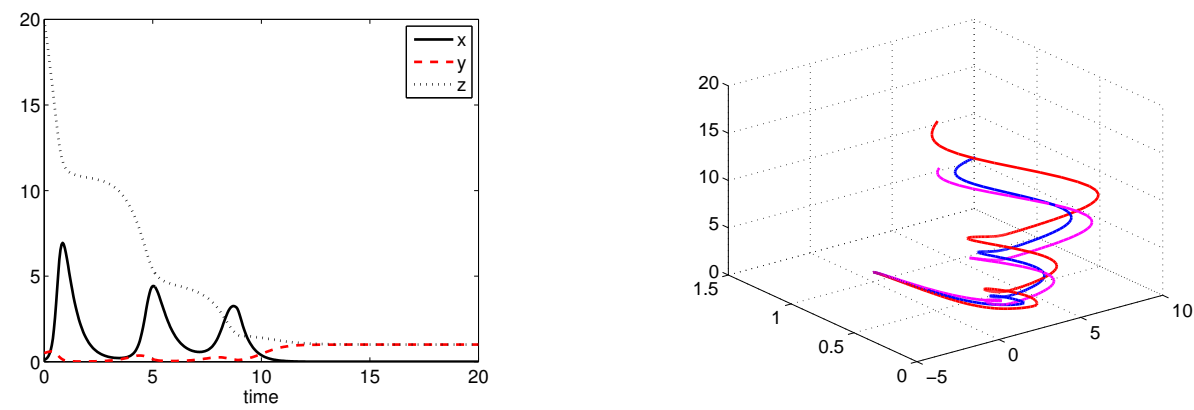

Fig. 4. Example of solution and phase space portrait for (2.1) in the case when the solution changes subregions of the phase space (parameter values: $a=15, b=2, c=2, d=1$, $e=1, f=3, k=1.5, m=1$ and $l=1)$

We have performed many simulations in which we have not observed the behaviour different from those described above and shown in Figs. 1-4. Typically, the solution to both models tends either to the positive steady state or to the semi-trivial one, even if it does not stay in the same subregion of the phase space for all time (see Fig. 4 where the solution passes from $\mathcal{A}_{2}^{1}$ to $\mathcal{A}_{1}^{1}$ ). If the maximal level of autotrophs (that is, their carrying capacity) is small, $M<b / a$, then there is no positive steady state for either (2.1) or (2.2), which leads to the extinction of heterotrophic organisms. If $M>b / a$, then there is a positive steady state for both systems, and typically, it is attractive. We interpret this case as the normal state which is observed in nature. The models confirm the commonly accepted view that people need to take care of green plants, especially trees in forests, which are necessary for us to survive.

Finally, we stress once again that in our opinion the modifications of the Dawidowicz and Zalasiński models proposed in [4] and in this paper are 
necessary. Without that modification, the level of oxygen can increase to infinity in some cases, which is impossible in nature.

4. Appendix. In this appendix we present the proofs of the properties of (1.2) similar to those proved in [4]. We mainly focus on the case of optimal level of oxygen, that is, $y<z<N$, in Lemmas 9 and 10, but we also study the case $z<y$ and $z<N$ in Lemma 11. The proofs of other properties are similar.

Lemma 9. If $b / a>M, e>c, d>f$, then for

$$
y_{0}<z_{0}<N-\frac{e}{c}\left(M-y_{0}\right)-x_{0} \frac{M}{b-a M}\left(\frac{e d}{c}-f\right)<N
$$

the solution to (1.2) stays in the subregion $\mathcal{D}_{1}^{2}=\{(x, y, z) \in \mathcal{D}: N>z>y\}$. Moreover, $(x, y, z) \rightarrow\left(0, M, z_{g}\right)$ where

$$
z_{g}=z_{0}+\frac{f}{d}\left(M-y_{0}\right)+\left(e-\frac{c f}{d}\right) \int_{0}^{\infty} y(s)(M-y(s)) d s .
$$

Proof. Consider the auxiliary variable $u(t)=z(t)-y(t)$. Under the assumptions of this lemma we have $u_{0}>0$. Calculating $\dot{u}=\dot{z}-\dot{y}$ one gets $\dot{u}=e y(M-y)-f x y-c y(M-y)+d x y=(d-f) x y+(e-c) y(M-y)>0$, under the assumed inequalities. Hence, $u$ is increasing, which implies $u(t)>0$ for every $t \geq 0$.

The solution to (1.2) stays in $\mathcal{D}_{1}^{2}$ only if the inequality $z(t)<N$ is satisfied. Using formula (1.3) one needs to estimate the integral $\int_{0}^{\infty} y(s)$ - $(M-y(s)) d s$. From the first equation of (1.2) we have $\dot{x}<(a M-b) x$. Hence, $x(t) \leq x_{0} \mathrm{e}^{-(b-a M) t}$ and $\dot{y} \geq c y(t)(M-y(t))-d x_{0} y(t) \mathrm{e}^{-(b-a M) t}$. This implies

$$
c y(t)(M-y(t)) \leq \dot{y}(t)+d x_{0} y(t) \mathrm{e}^{-(b-a M) t} .
$$

Therefore,

$$
\begin{aligned}
\int_{0}^{t} y(s)(M-y(s)) d s & \leq \frac{1}{c} \int_{0}^{t} \dot{y}(s) d s+\frac{d x_{0}}{c} \int_{0}^{t} y(s) \mathrm{e}^{-(b-a M) s} d s \\
& <\frac{M-y_{0}}{c}+\frac{d x_{0} M}{c(b-a M)} .
\end{aligned}
$$

Finally,

$$
z(t)<z_{0}+\frac{e}{c}\left(M-y_{0}\right)+x_{0} \frac{M}{b-a M}\left(\frac{e d}{c}-f\right)<N \quad \text { for every } t \geq 0,
$$

under the assumption of this lemma. 
The last part of the lemma is obvious if we know that the integral $\int_{0}^{\infty} y(s)(M-y(s)) d s$ converges. But $\int_{0}^{t} y(s)(M-y(s)) d s$ is increasing in $t$ and bounded above. Hence, it converges. This completes the proof.

LEMMA 10. If $b / a<M, x_{0}<(e / f)\left(M-y_{0}\right), z_{0}<N, e>c$ and $d>f$, then $(x, y) \rightarrow((c / d)(M-b / a), b / a)$ and $z$ is increasing such that the solution to (1.2) leaves $\mathcal{D}_{1}^{2}(z>N$ at some time $)$.

Proof. We know that the $(x, y)$ coordinates of the solution tend to the positive steady state of (1.2), which means $y \rightarrow b / a$ and $x \rightarrow(c / d)(M-b / a)$. As in the proof of Lemma 9, we easily show that $z$ is increasing until $z(t)<N$. We only need to show that it increases to $\infty$, which means that the solution leaves $\mathcal{D}_{1}^{2}$. For any $\varepsilon>0$ and sufficiently large $t$, that is, for $t>\bar{t}$, we have $b / a-\varepsilon<y(t)<b / a+\varepsilon$. Hence,

$$
\begin{aligned}
z(t) & >z_{0}+\frac{f}{d}\left(\frac{b}{a}-\varepsilon-y_{0}\right)+\left(e-\frac{c f}{d}\right) \int_{\bar{t}}^{t}\left(\frac{b}{a}-\varepsilon\right)\left(M-\frac{b}{a}-\varepsilon\right) d s \\
& >\frac{f}{d}\left(\frac{b}{a}-\varepsilon-y_{0}\right)+\left(e-\frac{c f}{d}\right)\left(\frac{b}{a}-\varepsilon\right)\left(M-\frac{b}{a}-\varepsilon\right)(t-\bar{t}) .
\end{aligned}
$$

Taking $\varepsilon=\frac{1}{2} \min \{b / a, M-b / a\}>0$ one gets $z(t) \rightarrow \infty$ as $t \rightarrow \infty$. This ends the proof.

LEMmA 11. If $M<N$, ed $<$ cf, $f<d$ and $y_{0}>(d / f) z_{0}$, then the solution to (1.2) stays in the subregion $\mathcal{D}_{4}^{2}=\{(x, y, z) \in \mathcal{D}: y>z$ and $z<N\}$.

If $z_{0}<y_{0}, z_{0}<N$ and $z_{0}+(e / c)\left(M-y_{0}\right)$, then the solution leaves $\mathcal{D}_{4}^{2}$.

Proof. If the solution stays in $\mathcal{D}_{4}^{2}$, then $x(t)$ can be expressed as a functional of $y$, similarly to $z(t)$, namely

$$
x(t)=\left(x_{0}+\frac{a}{d} y_{0}\right) \mathrm{e}^{-b t}-\frac{a}{d} y(t)+\frac{a}{d} \int_{0}^{t} y(s)(c M+b-y(s)) \mathrm{e}^{b(s-t)} d s .
$$

Therefore, if the solution stays in $\mathcal{D}_{4}^{2}$ and $y(t)$ has a limit, then $x(t)$ and $z(t)$ also have limits. Moreover, all derivatives $\dot{x}(t), \dot{y}(t)$ and $\dot{z}(t)$ have limits. This implies $x \rightarrow 0, y \rightarrow M$ and $z \rightarrow z_{0}+(e / c)\left(M-y_{0}\right)$.

Under the first list of assumptions we have $N>M>y_{0}>(d / f) z_{0}>z_{0}$ and $y(t)<M<N$ for every $t>0$, obviously. Hence,

$$
z(t)=z_{0}-\frac{f}{d} y_{0}+\frac{f}{d} y(t)+\frac{e d-c f}{d} \int_{0}^{t} y(s)(M-y(s)) d s<\frac{f}{d} y(t)<y(t)
$$

for $t>0$. This implies that the solution stays in $\mathcal{D}_{4}^{2}$.

Under the second list of assumptions we have $\lim _{t \rightarrow \infty} z(t)>b / a$, and therefore $z(t)>b / a$ for sufficiently large $t$, that is, $t>\bar{t}$. This implies 
$\dot{x}(t)>0$ for large $t$. Hence, $x(t)>x(\bar{t})$ for $t>\bar{t}$ and $x(t)$ does not tend to 0 . Thus, the solution leaves $\mathcal{D}_{4}^{2}$.

Acknowledgements. The work of UF was partially supported by the Polish Ministry of Science, grant No. 1P03A 02830.

\section{References}

[1] A. L. Dawidowicz and J. L. Zalasiński, On the mathematical model of morphology of leaves, in: Proc. 14th National Conf. on Applications of Mathematics in Biology and Medicine (Zawoja, 2005), 97-100.

[2] -, 一, A model of participation of autotrophic and heterotrophic organisms in phenomenon of life, in: Proc. 12th National Conf. on Applications of Mathematics in Biology and Medicine (Koninki, 2006), 37-39.

[3] U. Foryś, Mathematics in Biology, WNT, Warszawa, 2005 (in Polish).

[4] U. Foryś and Z. Szymańska, Analysis of heterotrophic-autotrophic organisms model, in: Proc. 13th National Conf. on Applications of Mathematics in Biology and Medicine (Serpelice, 2007), 43-48.

[5] J. D. Murray, Mathematical Biology. 1. An Introduction, Springer, New York, 2002.

[6] P. F. Verhulst, Notice sur la loi que population suit dans son accroissement, Corr. Math. Phys. 10 (1838), 113-121.

Urszula Foryś

Zuzanna Szymańska

Institute of Applied Mathematics and Mechanics

ICM

Faculty of Mathematics, Informatics and Mechanics

University of Warsaw

University of Warsaw

Pawińskiego 5a

Banacha 2

02-097 Warszawa, Poland

E-mail: urszula@mimuw.edu.pl

02-106 Warszawa, Poland

E-mail: mysz@icm.edu.pl 